\title{
Laxative Poisoning in Newborn Clinical Evolution Report of a Case
}

\author{
Alicia Santa Cortes González*, Vania Zamara Preciado Sánchez and Claudia Serine Fonseca \\ Universidad Veracruzana, Mexican Social Security Institute of Xalapa Veracruz, Mexico
}

Received: February 26, 2018; Published: March 08, 2018

*Corresponding author: Alicia Santa Cortés González, Universidad Veracruzana, Pediatrics Service General Hospital of Zona num.11 Mexican Social Security Institute of Xalapa Veracruz, Mexico, Tel: 2288185555; Email: aliciasantac@hotmail.com

\begin{abstract}
Introduction: Constipation is a common problem and is one of the main causes of consultation in the pediatric age, and the problems are indicated in the diagnosis, in the treatment, or in both. Its estimated prevalence varies between $0.3 \%$ and $8 \%$ of the child population. There is a case of male 1 month and 18 days of extra uterine life that are caused by the excessive use of laxatives of osmotic type associated with the use of stimulant laxatives in prolonged form and at high doses at a weight level, their clinical evolution and the secondary to this use. Conclusion: The use of laxatives from birth without having an adequate diagnosis of the cause of constipation, means that its use is indiscriminately causing alterations at the gastrointestinal level such as electrolyte imbalance and hypovolemic shock that can evolve to death if not timely manner.
\end{abstract}

Keywords: Constipation; Laxatives; Hydroelectric Imbalance

\section{Introduction}

Constipation is a common problem and is one of the main causes of consultation in the pediatric age; its complications indicate a problem in the diagnosis, in the treatment, or in both. Its estimated prevalence varies between $0.3 \%$ and $8 \%$ of the child population. It is also a reason for frequent consultation can represent $3-5 \%$ of visits to the pediatrician and up to $25 \%$ of children sent to the pediatric gastroenterology clinic [1]. There seems to be an increase in the frequency of constipation in recent years that could be related, among other factors, with a lower consumption of fiber in the diet and a more sedentary lifestyle, common characteristics of Western countries [2].

\section{Clinical Case}

Male 1 month 18 days of extra uterine life. It is a product of gestation 1 obtained by cesarean section due to acute fetal distress at 37 weeks of gestation, qualified with Agar 8/10. Mother of 35 years old housewife, father of 49 years of age without drug addiction. With medium socioeconomic environment, is fed to the mother's breast and supplemented with milk formula. From its birth it presents intestinal constipation that was handled by a private doctor with cisapride, dimethicone, loratadine and but ylhioscine due to colic, without improvement. Lacunose is added, and sodium picosulfate at high doses for a long time (3 weeks), micro lax is added, both laxatives at the same time. As well as iron, which, when interacting with cisapride, interferes with the absorption, so it was suspended. Continued with the indicated medications plus milk formula diluted to $50 \%$, with poor evolution.

He was taken to the emergency department due to poor general condition, dehydration with hypovolemic shock, dilated pupils with poor response, hypoactive, hypo reactive, hypo dynamic, marble, Glasgow for infants 3 of 15 with absent pulses and capillary refill greater than 5 seconds, with weak respiratory effort and heart rate less than 60 beats per minute, saturating less than 50\% oxygen, with capillary glycerin less than $30 \mathrm{mg} / \mathrm{dL}$, with hypo perfusion data, with difficult venous access, so emergency venodisection is performed right pelvic member. Initiated advanced management of cardiopulmonary resuscitation with administration of adrenaline, $0.2 \mathrm{mcg} / \mathrm{kg} / \mathrm{min}$. Cardiac massage, end tracheal intubation, with saline loads at $20 \mathrm{ml} / \mathrm{kg} /$ dose, twice with recovery of heart rate and saturation greater than $80 \%$ at 5 minutes. Management is started with dobutamine at $10 \mathrm{mcg} / \mathrm{kg} / \mathrm{min}$. Arterial blood gas was taken with a report of decompensate metabolic acidosis.

Alkalizing solutions and assisted ventilation are started, the laboratory report with hemoglobin anemia of $11.2 \mathrm{~g} / \mathrm{dL}$, hematocrit of $33 \%$, platelets $40510^{\wedge} 3 / \mu$ l, leukocytes $11.510^{\wedge} 3$ / $\mu$ l, neutrophils $5.410^{\wedge} 3$ / $\mu$ l, glucose $17 \mathrm{mg} / \mathrm{dL}$, urea $91.8 \mathrm{mg}$ / $\mathrm{dL}$, keratinize $0.7 \mathrm{mg} / \mathrm{dL}$, total protein $3.4 \mathrm{~g} / \mathrm{dL}$, chlorine $94 \mathrm{mmol}$ 
/ L, hypokalemia 2.56mmol / L, hyponatremia $122 \mathrm{mmol}$ / L, with elongated coagulation times TP $32.1 \mathrm{sec}$, TPT $40.2 \mathrm{sec}$. Correction of hydroelectrolyte imbalance, hypoglycemia and coagulation times with better evolution are initiated. It presents seizures secondary to hypoxemia and hyponatremia. During his stay he was managed in intensive therapy, presenting pneumonia, at elect sis and data of venous thrombosis in the right pelvic member, managed with enoxaparin with good response, he has a good evolution being discharged a month after being hospitalized. He has currently studied with pneumonia secondary to type 2 laryngomalacia and bronchial intermediate stenos is of $20 \%$, seizures secondary to hypoxic-ischemic encephalopathy, treated with diphenylhydantoin, lactose intolerance was ruled out by laboratory as a cause of chronic constipation, in control by medicine physical and rehabilitation due to slight motor delay with good evolution.

\section{Discussion}

An organic cause of constipation can rarely be established, in $90 \%$ of cases it corresponds to the functional cause. Although the physiopathology in childhood is not well known, in most cases we find a history of painful defecation that marks the beginning of the picture. The factors that may be associated with functional constipation are, in infants, weaning and the onset of ablation; in the preschool the problems during toilet training; in the schoolchildren the entrance to the school, the retention of the evacuation by the prohibition to go to the bathroom, emotional problems and changes of environment, as well as the little intake of fiber and water in the $\operatorname{diet}[3,4]$.

In these children, symptoms and warning signs should be evaluated to differentiate between functional constipation and secondary constipation [5]. In this patient the absence of evacuations was not documented more than twice a week, as established by one of the criteria of roam III, only for 36 hours of absence treatment was initiated with laxatives of osmotic type and lubricants without improvement associated with the indiscriminate use of stimulanttype laxatives, diphenylmethane derivatives such as sodium picosulfate for a long time and with overdose. The use of stimulant laxatives is only recommended for short periods of treatment. Its use in infants is not recommended. Prokinetics such as cisapride have also been used to treat constipation in children. Although it is superior to placebo, its use is not recommended considering that the benefits do not outweigh the risks [6]. Newborns exclusively fed with breastfeeding must explain to the mother the existence of a neurological immaturity of the colon, which causes the slowness of the evacuations, and that resolves spontaneously. If they are fed infant starter formulas, they should rule out intolerance to cow's milk (to protein or sugars), and change the formula. Between the four and six months the ablactación begins (introduction of foods in the diet), with fruits that improve the evacuations.
Glycerin suppositories are indicated in cases of newborns or infants who have 24 hours or more without evacuation, with symptoms of intestinal discomfort and distension, its application relaxes the rectal sphincter and allows expelling the accumulated fecal material in the rectal ampoule, with which the symptoms give. Likewise, clinical trials have been carried out in which it is mentioned that in patients with chronic functional constipation without response to adequate treatment, a protein elimination diet (extensive hydrolysates) can be used in children with a history of allergic processes. Sodium picosulfate is a drug that is hydrolyzed to its free form by bacterial enzymes, so it becomes active in the colon after the bacterial flora transforms it into the same active molecule of diphenylmethane by altering the net transport of liquids and electrolytes and has a direct promoter effect of intestinal motility, the most common side effects are colicky pain, bloating and electrolyte imbalance [7]. There is an interaction between the group of medicines such as cisapride and stimulant laxatives, administered at a higher dose than the weight and for a long time. What is causing electrolyte imbalance, seizures and dehydration that was what happened in this patient.

\section{Conclusion}

The treatment of constipation in children should be done based on functional clinical diagnosis as the main cause in pediatrics, being necessary to discard clinical data that suggest underlying organic problem, infants younger than 6 months often have dyskinesia, which is defined according to the criteria of roam III as the presence of a) pushing and crying for at least 10 minutes before achieving the evacuation of soft stools and b) lactating without any health problem. This disorder does not merit treatment. In this work there are no conflicts of interest or some type of financing. Informed consent was made in this patient.

\section{References}

1. Mondragón Luis De la Torre, Hernández Vez Gabriela (2014) Functional constipation in pediatrics. Acta, Pediátr Mex 35(5): 411-422.

2. Sánchez Ruiz Francisco, Gilbert Juan José, Bedate Calderón, Espín Jaime (2010) Diagnostic-therapeutic protocols of Gastroenterology, Hepatology and Pediatric Nutrition SEGHNP- AEP.

3. Waiter Salces C, Rizo Pascual JM (2011) Functional constipation of the National Health System. In Ter Sist Nac Salud 35:13-20.

4. Bautista Casasnovas Adolfo L (2011) Chronic Constipation. An Pediatr Contin 9(4): 201-208.

5. Remes Troche JM, Gómez Escudero O, Icaza Chávez ME, Noble Lugo A, López Colombo A, et al. (2011) Diagnostic guides and treatment of constipation in Mexico. Medical and surgical treatment. Journal of Gastroenterology of Mexico 76(2): 141-154.

6. Paré P, Fedorak RN (2014) Systematic Review of Stimulant and Nonstimulant Laxatives for the Treatment of Functional Constipation. Canadian Journal of Gastroenterology and Hepatology 28(10): 549-557. 


\section{(c) () This work is licensed under Creative}

Submission Link: https://biomedres.us/submit-manuscript.php

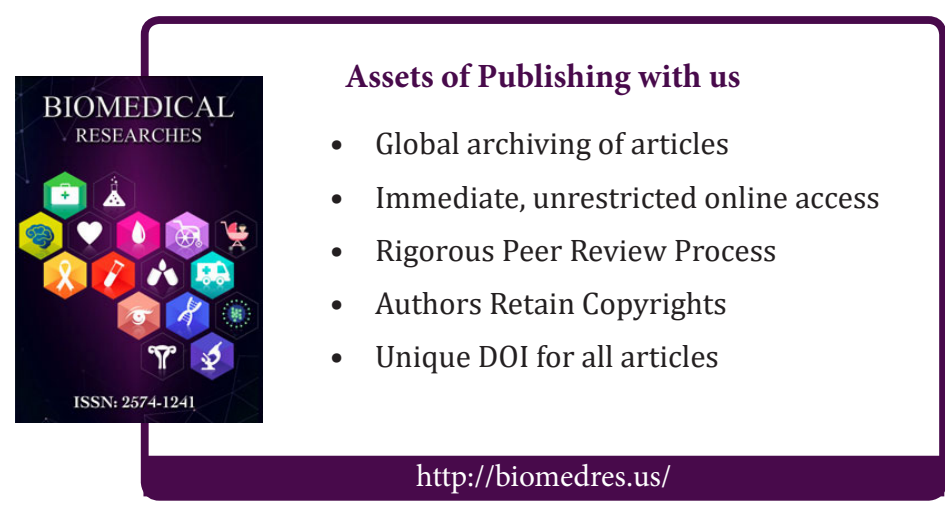

